\title{
A census of the physical parameters of nearby galaxies
}

\author{
Anna Gallazzi ${ }^{1,2}$, J. Brinchmann ${ }^{3}$, S. Charlot ${ }^{4}$ \\ and S. D. M White ${ }^{2}$ \\ ${ }^{1}$ Max-Planck-Institut für Astronomie, Königstuhl 17, D-69117 Heidelberg, Germany \\ email: gallazzi@mpia-hd.mpg.de \\ ${ }^{2}$ Max-Planck-Institut für Astrophysik, Karl-Schwarzschild-Str. 1, D-85748 Garching bei \\ München, Germany \\ ${ }^{3}$ Centro de Astrofísica da Universidade do Porto, Rua das Estrelas - 4150-762 Porto, Portugal \\ ${ }^{4}$ Institut d'Astrophysique du CNRS, 98 bis Boulevard Arago, F-75014 Paris, France
}

\begin{abstract}
We combine together physical parameters estimates for a large sample of galaxies drawn from the Sloan Digital Sky Survey Data Release Two (SDSS DR2), spanning wide ranges in galaxy properties, in order to provide a census of the physical parameters of galaxies in the local Universe. Stellar metallicities, masses and ages for this sample have been estimated comparing the strengths of selected absorption features in each observed spectrum to a comprehensive Monte Carlo library of star formation histories, following a Bayesian statistical approach. This allows us also to derive the total amount of metals locked up in stars today, and hence the average stellar metallicity of galaxies in the local Universe, finding a value of $1.03_{-0.15}^{+0.14} Z_{\odot}$. We also quantify the relative contribution to the total densities of mass and metals in stars from galaxies with different properties.
\end{abstract}

Keywords. galaxies: formation, galaxies: evolution, galaxies: stellar content

\section{Introduction}

Accurate estimates of the mass, mean age and chemical composition of the stellar populations in galaxies are crucial to study on one side the past history of star formation and chemical enrichment of different galaxy types, and on the other side the evolution over cosmic time of the ensemble star formation and chemical enrichment rates.

Extracting physical parameters estimates from galaxy spectra requires comparison with population synthesis models. In recent years new models have become available based on stellar libraries at higher spectral resolution and with a more uniform coverage of the stellar parameters (e.g, Vazdekis 1999, Bruzual \& Charlot 2003, hereafter BC03), with respect to previous models. These improvements permit to extend the analysis to young stellar populations. Based on such models and with the advent of large spectroscopic surveys, several methods have been developed to extract estimates of the physical parameters of galaxies with any star formation history $(\mathrm{SFH})$, either from their whole (optical) spectra (Panter et al. 2003, Cid Fernandes et al. 2005) or from individual absorption features, such as those defined in the Lick system, measured in the same way in the model and observed spectra (Gallazzi et al. 2005, hereafter G05).

At $z=0$, the large statistics provided by modern spectroscopic surveys and the new physical parameters estimates make it possible to confront the location of galaxies with different SFHs in the parameter space defined by stellar mass, metallicity and age (e.g. G05). The observed bimodal distribution of galaxy optical colours have been translated into a bimodality in physical and morphological parameters: a smooth transition occurs 
at a critical stellar mass of $3 \times 10^{10} \mathrm{M}_{\odot}$ from disk-dominated, young, metal-poor, gas-rich galaxies to bulge-dominated, old, metal-rich, gas-poor galaxies (e.g. Kauffmann et al. 2003, Kannappan et al. 2004, G05). Early-type galaxies, belonging mainly to the latter class, follow tight scaling relations linking their mass to the properties of their stellar populations. Recent works have quantified both the variations in age, metallicity and element abundance ratios along the observed relations, and the mass-dependent scatter in age and metallicity about them (e.g. Thomas et al. 2005, Gallazzi et al. 2006). As far as star-forming galaxies are concerned, it has been shown that stellar and gas-phase metallicity exhibit a similar dependence on stellar mass and correlate with each other with approximately the expected unit slope (Cid Fernandes et al. 2005, G05).

In this contribution we address the following questions: given the relative weight of galaxies in the metallicity-age-mass relations, what is the resulting average stellar metallicity? What is the total amount of metals and mass locked up into stars by the present epoch? Which is the fractional contribution of different galaxy types to these quantities? The total amount of metals and mass produced and locked up in stars constitutes the fundamental constraint at redshift zero to the cosmic star formation history.

\section{The sample: individual and coadded spectra}

The sample we use is based on 164,746 unique SDSS DR2 (Abazajian et al. 2004) spectra of galaxies with Petrosian $r$-band magnitudes in the range $14.5 \leqslant r \leqslant 17.77$, and with $0.005<z \leqslant 0.22$. All galaxy types are included, from star-forming late-type to quiescent early-type galaxies.

As explained in more detail in G05, we have obtained median-likelihood estimates of stellar metallicity $\left(Z_{*}\right)$, stellar mass $\left(M_{*}\right)$ and $r$-band light-weighted age $\left(t_{r}\right)$ for this sample, by comparing each spectrum to a Monte Carlo library of BC03 models, covering the full range of possible SFHs. The comparison is based on five spectral absorption features, namely $\mathrm{D} 4000, \mathrm{H} \beta, \mathrm{H} \delta_{A}+\mathrm{H} \gamma_{A},\left[\mathrm{Mg}_{2} \mathrm{Fe}\right]$ and $[\mathrm{MgFe}]^{\prime}$, all of which have at most a weak dependence on element abundance ratios.

Individual galaxy spectra are used only if they have a mean signal-to-noise ratio per pixel $(\mathrm{S} / \mathrm{N})$ of at least 20 , required to derive reliable stellar metallicity estimates. This requirement is met by 25 percent of the original sample. The remaining 75 percent of galaxies (mainly low surface brightness galaxies) is included by coadding their spectra to derive average high-S/N spectra. We divide low-S/N galaxies into bins of velocity dispersion, $r$-band absolute magnitude and D4000 (in order to guarantee small scatter in physical parameters). In each such bin we coadd galaxy spectra weighting each individual low-S/N spectrum by $1 / \mathrm{V}_{\max }$, where $\mathrm{V}_{\max }$ is the maximum visibility volume given by the bright and faint magnitude limits of the survey. The physical parameters derived from the stacked spectra (with the same Bayesian analysis as for individual galaxy spectra) can be interpreted as the $\left(1 / \mathrm{V}_{\max }\right)$-weighted average parameters of the galaxies that contribute to each coadded spectrum. The stacking technique allows us to retrieve the physical parameters of galaxies with low-S/N spectra with a much greater accuracy than what we could do from their original spectra. (The technique and the results of the next section will be discussed in more details in a forthcoming paper.)

\section{Results}

\subsection{The total stellar metallicity in the local Universe}

Combining information for the individual high-S/N galaxies and the coadded spectra of low-S/N galaxies, and weighting each galaxy by $w=1 / \mathrm{V}_{\max }$, we estimate the stellar mass 
density $\left(\rho_{*}\right)$ and the mass density of metals in stars $\left(\rho_{Z}\right)$ of the local Universet:

$$
\begin{gathered}
\rho_{Z}=\sum_{i}\left(Z_{*, i} M_{*, i} w_{i}\right)=8.254_{-1.94}^{+2.53} \times 10^{6} h_{70} \mathrm{M}_{\odot} \mathrm{Mpc}^{-3} \\
\rho_{*}=\sum_{i}\left(M_{*, i} w_{i}\right)=4.013_{-0.97}^{+0.67} \times 10^{8} h_{70} \mathrm{M}_{\odot} \mathrm{Mpc}^{-3}
\end{gathered}
$$

These valuesł agree with different observational estimates (e.g. Fukugita et al. 1998, Glazebrook et al. 2003 for the stellar mass, Balogh et al. 2001, Finoguenov et al. 2003, Huang et al. 2003 for the stellar metallicity) and with predictions from chemo-photometric models of galaxies (e.g. Calura \& Matteucci 2004).

Combined together, equations 3.1 and 3.2 give a (mass-weighted) average metallicity in stars in the local Universe of:

$$
\left\langle Z_{*}\right\rangle=\rho_{Z} / \rho_{*}=1.03_{-0.15}^{+0.14} Z_{\odot}
$$

As pointed out by Edmunds 1999, a current average stellar metallicity of about solar might be expected if most of the baryons have been locked up in stars by the present epoch, assuming standard yields. Stars are certainly not the only reservoir of metals, but they host presumably the majority of metals today (more than 50 percent, e.g. Davé \& Oppenheimer 2006).

\subsection{An inventory of the stellar metallicity and stellar mass}

Fig. 1 compares the distribution of stellar metallicity (shaded histograms) to the distribution of stellar mass (solid lines) as a function of different galaxy properties: stellar mass

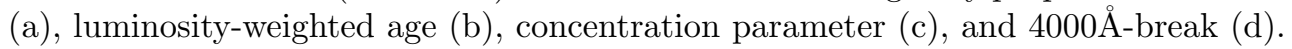

The picture read from Fig. 1 is in agreement with the mass-metallicity relations for early- and late-type galaxies. Half of the metals come from galaxies more massive than $\sim 10^{11} \mathrm{M}_{\odot}$ ( slightly higher than the transition mass of $\left.3 \times 10^{10} \mathrm{M}_{\odot}\right)$, with concentration parameter greater than 2.6 (slightly lower than our adopted threshold of 2.8 to select bulge-dominated galaxies), and with fairly old stellar populations ( $t_{r} \sim 6 \mathrm{Gyr}$ ). These galaxies contribute about 40 percent of the total stellar mass density. Conversely, galaxies with stellar masses below the transition mass, concentration parameters typical of disk-dominated galaxies and with predominantly young stellar populations contribute 25 percent of metals (half of those hosted by massive galaxies) and about 35 percent of stellar mass (a fraction similar to that contributed by massive galaxies). Finally, it is interesting to notice that both the stellar mass and the stellar metallicity distribution peak at a mean age of slightly less than 9 Gyr, which corresponds to a redshift of about 1.3. This falls in the redshift range where the cosmic SFH starts to decline (e.g. Lilly et al. 1996, Madau et al. 1998).

$\dagger$ We assume $\Omega_{\Lambda}=0.7, \Omega_{M}=0.3, \mathrm{H}_{0}=70 \mathrm{~h}_{70} \mathrm{~km} \mathrm{~s}^{-1} \mathrm{Mpc}^{-1}$, and the Chabrier 2003 initial mass function.

$\ddagger$ The quoted systematic uncertainties take into account the following potential sources of bias: 1) our stacking approach to include low-S/N galaxies; 2) variations in element abundance ratios, not included in BC03 models; 3) aperture effects; 4) the prior assumed to generate the Monte Carlo library of SFHs; 5) normalization of the mass-to-light ratios using Petrosian magnitude compared to model magnitude (our default). 


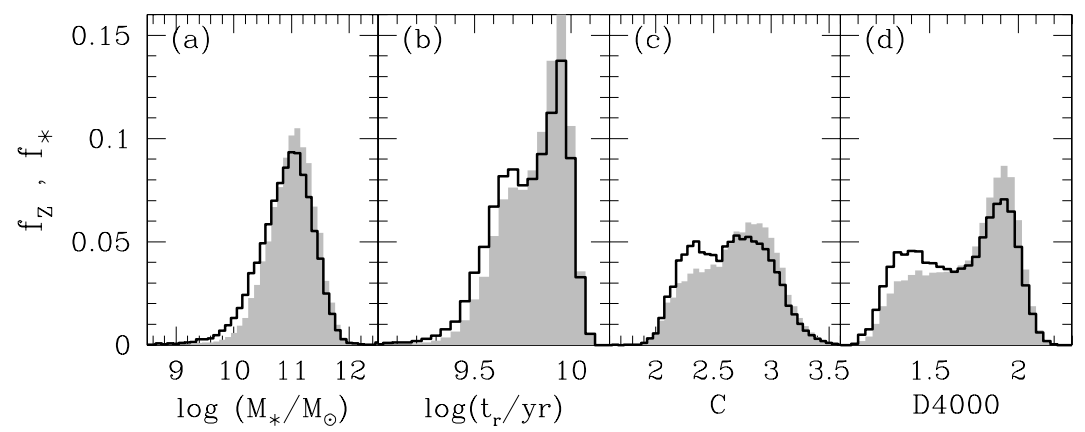

Figure 1. Fraction of metals ( $f_{Z}$, shaded histogram) and of mass $\left(f_{*}\right.$, solid line) locked up in stars as a function of various galaxy properties: stellar mass (a), r-band luminosity weighted age (b), concentration parameter (c), 4000- $\AA$ break strength (d).

\section{Acknowledgements}

AG acknowledges support by the DFG's Emmy Noether Program. JB acknowledges the receipt of FCT grant SFRH/BPD/14398/2003. Funding for the creation and distribution of the SDSS Archive has been provided by the Alfred P. Sloan Foundation, the Participating Institutions, the National Aeronautics and Space Administration, the National Science Foundation, the U.S. Department of Energy, the Japanese Monbukagakusho, and the Max Planck Society. The SDSS Web site is http://www.sdss.org/.

\section{References}

Abazajian, K., et al. 2004, AJ, 128, 502

Balogh, M. L., Pearce, F. R., Bower, R. G., \& Kay, S. T., 2001, MNRAS, 326, 1228

Chabrier, G., 2003, PASP, 115, 763

Bruzual, G., \& Charlot, S., 2003, MNRAS, 344, 1000

Calura, F., \& Matteucci, F., 2004, MNRAS, 350, 351

Cid Fernandes, R., Mateus, A., Sodré, L., Stasińska, G. \& Gomes, J. M., 2005, MNRAS, 358,363

Davé R. \& Oppenheimer, B. D., 2007, MNRAS, 374, 427

Edmunds, M. G., 1990, MNRAS, 246, 678

Finoguenov, A., Burkert, A., \& Böhringer H., 2003, ApJ, 594, 136

Fukugita, M., Hogan, C. J., \& Peebles, P. J. E., 1998, ApJ, 503, 518

Gallazzi, A., Charlot, S., Brinchmann, J., White, S. D. M., \& Tremonti, C. A., 2005, MNRAS, 362,41

Gallazzi, A., Charlot, S., Brinchmann, J., \& White, S. D. M., 2006, MNRAS, 370, 1106

Glazebrook, K., et al. 2003, ApJ, 587, 55

Huang, J.-S., Glazebrook, K., Cowie, L. L., \& Tinney, C., 2003, ApJ, 584, 203

Kannappan, S. J., 2004, ApJ, 611, L89

Kauffmann, G., et al. 2003, MNRAS, 341, 33

Lilly, S. J., Le Fevre, O., Hammer, F., \& Crampton, D., 1996, ApJ, 460, L1

Madau, P., Pozzetti, L., \& Dickinson, M., 1998, ApJ, 498, 106

Panter, B., Heavens, A., \& Jimenez, R., 2003, MNRAS, 343, 1145

Thomas, D., Maraston, C., Bender, R., \& de Oliveira, C. M., 2005, ApJ, 621, 673

Vazdekis, A., 1999, ApJ, 513, 224 


\section{Discussion}

Peletier: I would like to complain to your statement that $\langle Z\rangle$ in the local Universe is close to solar. Since stellar evolutionary models give absolute ages which have a considerable systematic errors, the metallicity scale will also have such an error. Also I personally would prefer if people would use the term 'luminosity weighted age' rather than 'light weighted age'.

GALLAzZI: Uncertainties in stellar evolutionary models are certainly a concern, especially considering the age-metallicity degeneracy that affects massive, bulge-dominated galaxies (we find that this degeneracy is less severe for lower mass galaxies). We consider several sources of systematic errors and we find that overall the systematic uncertainty can be up to $\sim 30 \%$. I would like to stress however that, although the age-metallicity degeneracy is still present on a galaxy-by-galaxy basis, it does not dominates the trends in metallicity versus mass and the distribution of galaxies in the mass-age-metallicity plane. Moreover working with the entire likelihood distribution (Bayesian statistic) allows us to quantify the extent of such degeneracy (confidence contours in age-metallicity distribution).

Cid-Fernandes: Do you include very low $Z_{*}$ models in your comparison library?. This is also related to the question of how do you choose a library of models, and the effects of your choice of this library?.

GaLLAzzi: Metallicity can vary over the whole range in metallicities provided by the BC03 models (i.e., from $2 \%$ up to $2.5 \times \mathrm{Z}_{\odot}$. We assume a "maximum-ignorance" prior in all the parameters defining the models in our library (i.e. uniform prior). However we allow for a smoothly decreasing density of models from $\mathrm{Z}=20 \% \mathrm{Z}_{\odot}$ down to $\mathrm{Z}=2 \% \mathrm{Z}_{\odot}$, because low-metallicity models are less plausible and less reliable. Certainly, in the Bayesian approach the choice of the underlying prior is a crucial point. It is important to check that the derived distributions in physical parameters is not dominated by the prior distribution and that the results are stable when changing some assumption.

DisneY: Comment: people should not forget SDSS is a $50 \mathrm{sec} 2 \mathrm{~m}$ telescope survey. It will not easily find LSB and dwarf galaxies except nearby. But nearby galaxies cannot be reduced at present because pipeline problems.

GaLLAzZI: The red selection and the homogenous sampling of the SDSS survey over large ranges in galaxy parameters space offers one of the sample of nearby galaxies most suited for performing a census of baryons and metals. It is true however that the survey misses the lowest surface brightness and dwarf galaxies. This should not be too much of a concern in this work: given the very low metallicities of such galaxies, which extend to low masses the mass-metallicity relation quantified in SDSS studies, their contribution to the total amount of metals is expected to be negligible. 